\title{
Development of an Autonomous Aquarium System for Maintaining Deep Corals
}

By Alejandro Olariaga, Andrea Gori, Covadonga Orejas, and Josep-Maria Gili

\section{INTRODUCTION}

Keeping deep corals alive under optimum water temperature and quality conditions is not an easy task. It is important to achieve a balance among a high-water renewal rate, constant water temperature, flow speed, and nutrient concentrations (especially ammonium). In trying to find a "meeting point" among all of these factors, the ZAE (Experimental Aquaria Area) of the Instituto de Ciencias del Mar (CSIC) in Barcelona developed an aquarium system that maintains constant temperature conditions in an open water circuit for five deep coral species being kept for HERMES project experimental purposes (Figure 1). The new system operates with a temperature variation of $\pm 0.2^{\circ} \mathrm{C}$, allowing culture conditions similar to those in the field.

\section{TECHNICAL DESCRIPTION AND SYSTEM FUNCTIONINC}

The aquarium system has been set up in a cool room at $12^{\circ} \mathrm{C}$, which is similar to the environmental temperature in the coral's natural habitat: 250-m water depth in the Cap de Creus Canyon, northwestern Mediterranean. Figure 2 shows the complete system, including all components and the connections between them. The system is managed through the temperature controller ( $\mathrm{T} 1$ ), which monitors the water temperature of the storage tank (TA1). An electric signal (S1) activates the electrical outlet $(A)$, which allows water input to the aquarium system. The temperature sensor in $\mathrm{T} 1$ has $0.1^{\circ} \mathrm{C}$ accuracy and a minimal hysteresis of $0.2^{\circ} \mathrm{C}$.
The seawater supply in the ZAE is at a higher temperature than is optimal for the corals; thus, the water temperature in TA1 increases. When water temperature inside TA1 increases $0.2^{\circ} \mathrm{C}$ (the hysteresis of the temperature sensor T1), the electrical outlet ( $A$ ) stops water flow into the aquaria.

The cool room temperature is controlled by a sensor (T2). The temperature inside the container is slightly lower than in the aquaria. In this way, the water temperature in the storage tank TA1 decreases, quickly equilibrating with the cool room. When TA1 temperature decreases by $0.2^{\circ} \mathrm{C}$, the electrical outlet is automatically activated, allowing inflow of water to the aquaria.

The storage water in TA1 is pumped to TD2 by the electrical pump P1. When the water volume in TD2 reaches its maximum level, water is transferred to TA1. TD2 has six taps (Qd, Qe, Qf, Qg, Qh, and Qi) that distribute the water to the different aquaria, taking into account that the sum of all six cannot exceed the flow of P1.

The water in each aquarium is recirculated through the submerged pumps (RP), which homogenize the water masses and generate currents-essential for the corals. To avoid overflow, each aquarium is equipped with an outlet to expel the excess water to TA2 when it exceeds the maximum level. In the same way, TA2 is provided with an outlet to eject excess water, as well as an electric pump (P2), which discharges the surplus water to TA1 after passing through a biological filter that eliminates suspended particles.

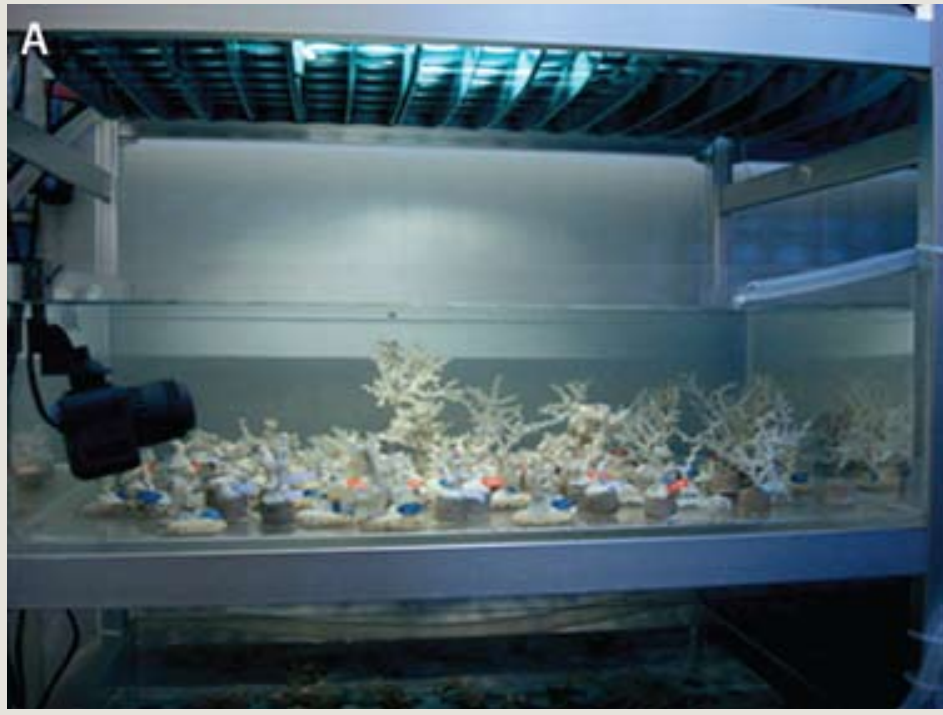

Figure 1. (A) Aquaria containing the cold-water corals Madrepora oculata and Lophelia pertusa, as part of the new autonomous system developed by ZAE (Instituto de Ciencias del Mar (CSIC), Barcelona). (B) Aquarium with corals Desmophyllum cristagalli (left) and Dendrophyllia cornigera (center).

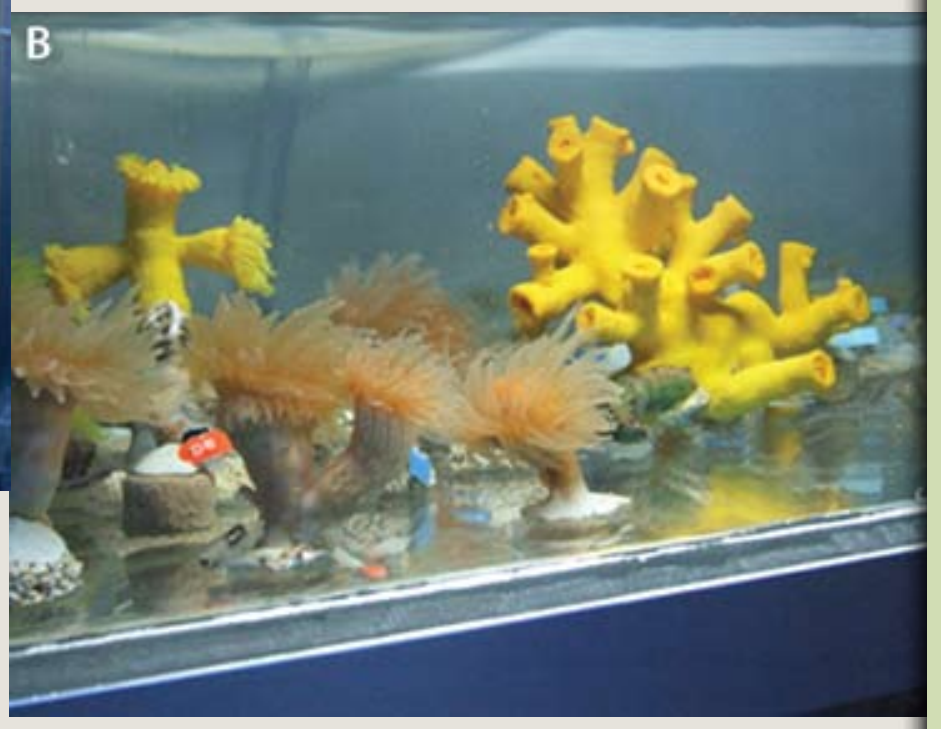


Thanks to the features of TA2, the system can function either as a closed system (water flowing between TA1 and TA2 through the aquaria) or as an open system (removing water from the system). This double function is achieved by the inversion of the electrical signal that controls the electronic outlet, thus allowing (1) overflow of excess when water is supplied to the system (signal S1), or (2) retention of water in the closed circuit (signal S2).

\section{ADDITIONAL FUNCTIONS}

The corals are fed every day. To make this task easier, a feeding timer is attached to the pump (P1) (see Figure 2). This timer cuts the water flow in each aquarium for a couple of hours each day in order to retain the food particles, allowing the organisms access to food during that time period. The feeding time should not exceed three hours because after this time, the ammonium level increases considerably and temperature decreases in the aquaria.

A supplementary function of the temperature controller is an alarm that can be set to activate the flow system when the temperature reaches the established maximum.

\section{ACKNOWLEDGEMENTS}

This work was supported by the European Project HERMES (EU contract 511234), the DEEP CORAL project (MEC, CTM 2005-07756-CO2-00), and the AC (CTM2007-28758-E). We thank P. Siles, X. Leal, and D. Pina for technical support. Thanks to L. Yebra for helping with the English version.

\section{Alejandro Olariaga (olariaga@icm.csic.es) is a technician in the Departa- ment de Biologia Marina i Oceanografia, Instituto de Ciències del Mar (CSIC), Barcelona, Spain. Andrea Gori is a graduate student in the Departament de Biologia Marina i Oceanografia, CSIC, Barcelona, Spain. Covadonga Orejas is Postdoctoral Scientist, Departament de Biologia Marina i Oceanografia, CSIC, Barcelona, Spain. Josep-Maria Gili is Research Professor and Assistant Director, Departament de Biologia Marina i Oceanografia, CSIC, Barcelona, Spain.}

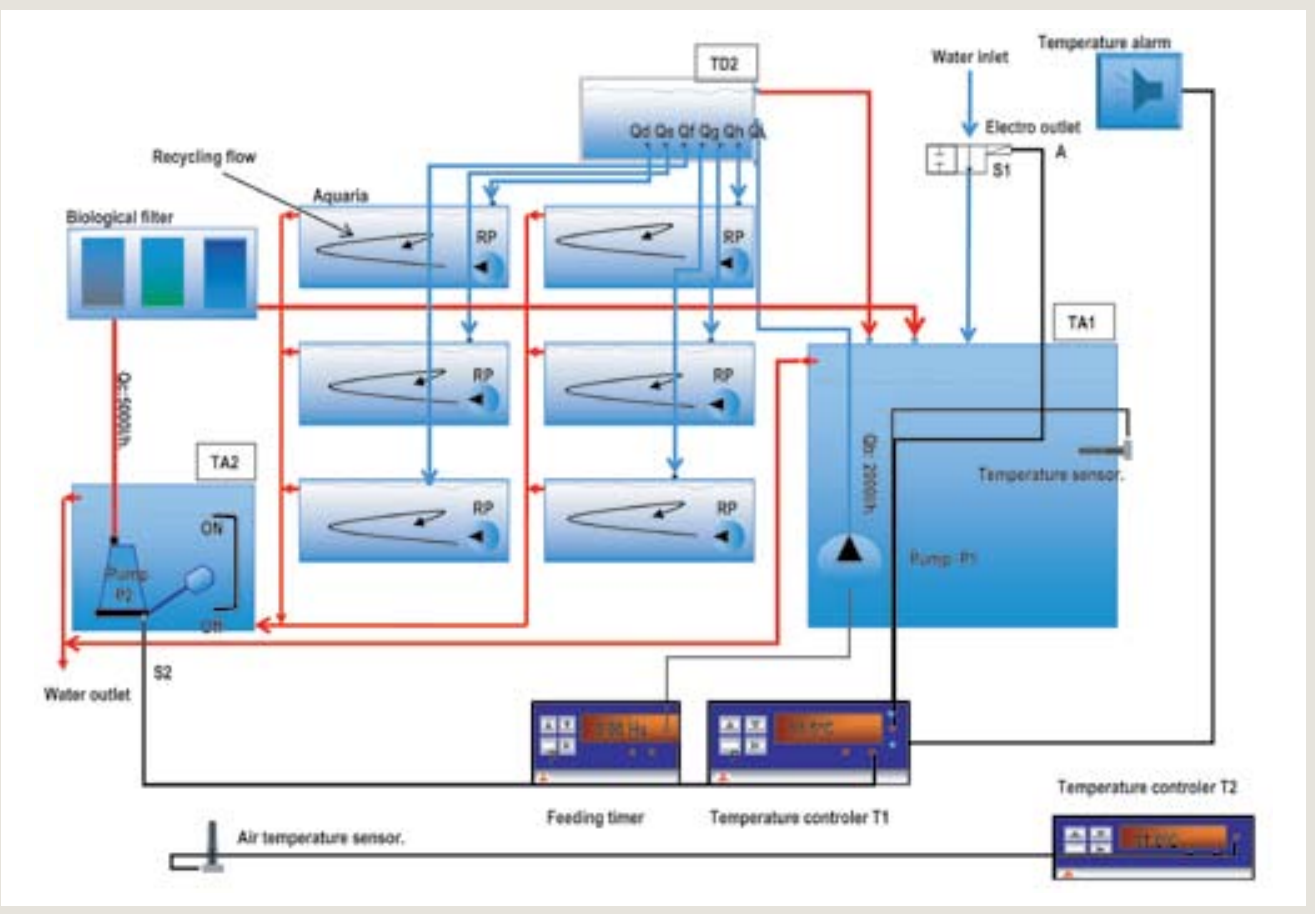

Figure 2. Scheme of the aquarium system, which consists of (1) an electrical outlet (A) that, when temperature activated, allows water to flow to (2) the 500-I storage tank TA1; water is pumped $\left(\mathrm{P} 1,2000 \mathrm{I} \mathrm{h}^{-1}\right)$ from TA1 to (3) tank TD2, which distributes the water to the six aquaria (76 liters each). The aquaria are each supplied with a recirculating pump (RP); outgoing water from each aquarium is collected in (4) tank TA2, supplied with a pump $\left(\mathrm{P} 2,5000 \mathrm{I}^{\mathrm{l}} \mathrm{h}^{-1}\right)$. This tank controls the "closed" or "open" circuit option: when closed, water is pumped to TA1; when open, water is pumped out of the system. $\mathrm{T} 1$ and $\mathrm{T} 2$ are the temperature controls for the seawater and for the container, respectively. A biological filter and a feeding timer are also displayed in the scheme. 